\title{
Effect of Nucleus Basalis Magnocellularis Lesions on Memory and Hippocampal Brain- Derived Neurotrophic Factor, IL-1 $\beta$, Glucose, and Corticosterone Levels in Adult Rats
}

\author{
Nasrin Hosseini ${ }^{1{ }^{2 *}}{ }^{(\mathbb{D}}$, Maryam Radahmadi ${ }^{2}$, Hojjatollah Alaei ${ }^{2}$, Shabnam Nadjafi ${ }^{1}$ \\ ${ }^{1}$ Neuroscience Research Center, Iran University of Medical Sciences, Tehran, Iran \\ ${ }^{2}$ Department of physiology, School of Medicine, Isfahan University of Medical Sciences, Isfahan, Iran
}

\begin{abstract}
Background: The nucleus basalis magnocellularis (NBM) sends projections to the hippocampus that are implicated in learning and memory formation. Despite ample evidence proposing that cognitive function impairment related to neurodegeneration, it may result from alteration of biochemical substances. We aimed to investigate the effects of NBM lesions on the hippocampal interleukin1 beta (IL-1 $\beta$ ), brain-derived neurotrophic factor (BDNF), and corticosterone levels, as inflammation markers, and hallmarks of neurodegeneration, stress, and metabolic status.

Methods: Thirty-six male Wistar rats were randomly put in control, sham, and NBM-lesioned groups. After inducing the lesion using an intra-NBM injection of $10 \mu \mathrm{g}$ ibotenic acid (5 $\mu \mathrm{g} / \mu \mathrm{L}$, each side) in rats, memory was estimated using the passive avoidance test. Moreover, serum and hippocampal $\mathrm{IL}-1 \beta$ levels, as well as the hippocampal corticosterone, BDNF, and glucose levels were measured after 42 days.

Results: Findings indicated a significant impairment of retention at different intervals in the NBMlesioned group. BDNF decreased whereas corticosterone, glucose, and IL-1 $\beta$ levels increased in the hippocampus. Also, the levels of serum IL-1 $\beta$, hippocampal BDNF, corticosterone, and glucose had significant correlations with hippocampal IL-1 $\beta$ levels.

Conclusion: The synchronous alterations of some hippocampal factors, including BDNF, corticosterone, IL-1 $\beta$, and glucose, caused by NBM lesion suggest that their interaction might play a significant role in neurodegeneration and relevant learning and memory impairments.

Keywords: Nucleus basalis magnocellularis; Hippocampus; Neurotrophic factor; Cytokine; Stress.
\end{abstract}

\section{*Correspondence to} Nasrin Hosseini,

Neuroscience Research Center, Iran University of Medical Sciences, Tehran, Iran. Tel: 0098-021-86704529; Fax: 0098-021-86704529; Email: hoseini.n@iums.ac.ir

Published online March 27, 2021

Citation: Hosseini N, Radahmadi M, Hojjatollah Alaei H, Nadjafi S. Effect of nucleus basalis magnocellularis lesions on memory and hippocampal brain-derived neurotrophic factor, il-1 $\beta$, glucose, and corticosterone levels in adult rats. Clin Neurosci J. 2021;8(2):67-75. doi:10.34172/icnj.2021.15.

\section{Introduction}

The basal forebrain neurodegeneration results in learning and memory impairment. The basal forebrain cholinergic neurons along with the non-cholinergic ones are distributed in the medial septal nucleus, diagonal band nuclei, nucleus basalis, and substantia innominata. Several cholinergic fiber projections are sent to the amygdala, neocortex, and hippocampus. ${ }^{1}$

Many studies have emphasized the effects of diabetes mellitus, stress, and inflammation on the onset and progression of memory impairment. Nevertheless, only some of these conditions have been thoroughly investigated..$^{2-4}$ Furthermore, these studies did not connect these abnormalities with a primary pathogenic mechanism related to the cognitive impairments; instead, they have merely proposed various theories and focused on a specific pathological condition.

Cortisol in humans or corticosterone (CORT) in rodents is the major stress hormone with many receptors in the hippocampus affecting its functions. ${ }^{2}$ An increase in both acute endogenous and exogenous glucocorticoids can lead to lack of motivation, attention deficit, and memory impairment. ${ }^{2,3}$ Similarly, stress and high-dose corticosterone are recognized to cause spatial memory deficits in rodents navigating the Morris Water Maze task. $^{5}$

There is an exceeding interest in finding the bidirectional associations between the immune system and the central nervous system (CNS). Accordingly, a high density of interleukin-1 (IL-1) type I receptors in the hippocampus ${ }^{6}$ was associated with memory consolidation impairment. ${ }^{7}$ Other research studies proposed the implication of IL$1 \beta$ in neurodegenerative events ${ }^{8}$ chronic inflammation, ${ }^{4}$ diabetes, ${ }^{9}$ inflammatory bowel disease, ${ }^{10}$ asthma, ${ }^{11}$ and autoimmune arthritis. ${ }^{12}$ Moreover, IL- $1 \beta$ may represent a modulatory role in the synaptic plasticity, neuroendocrine,

(C) 2021 The Author(s). This is an open access article distributed under the terms of the Creative Commons Attribution License (http:// creativecommons.org/licenses/by/4.0/), which permits unrestricted use, distribution, and reproduction in any medium, provided the original work is properly cited. 
and neurobehavioral stress responses. ${ }^{13,14}$

Furthermore, the modulatory effects of IL- $1 \beta$ on brainderived neurotrophic factor (BDNF) are important for memory consolidation ${ }^{15}$ but their relationship with other mediators remains to be further investigated. Also, neurotrophins like BDNF may be involved in the survival, recovery, and protection of healthy and damaged neurons. Some studies demonstrated the role of BDNF and pro-BDNF in synaptic plasticity, memory processing, and storage ${ }^{16,17}$ through ERK2 signaling pathways in the dentate gyrus and perirhinal cortex. Besides, lack of hippocampal BDNF gene has been associated with impaired spatial learning and novel object recognition. ${ }^{18}$ In total, results of former studies suggested that aging and inflammation can exacerbate the hippocampal inflammatory responses, ${ }^{6}$ decrease BDNF levels, ${ }^{15}$ and may also cause long-term memory deficits.

Other studies have focused on diabetes mellitus as a condition that alters the peripheral and central glucose metabolism. Glucose hemostasis alteration in the brain has important impacts on $\mathrm{AD}$ symptoms. ${ }^{19}$ The brain needs a high energy level for generating action potentials, producing neurotransmitters, and cell signaling. ${ }^{19}$ Most importantly, glucose homeostasis can directly affect the memory function in humans as well. In addition to the direct effects of glucose on neuronal metabolisms, insulin and IGF-I may indirectly affect the brain metabolism; hence, it may activate some phosphatidylinositol trisphosphate kinase pathways (e.g., Ras-MAPK-ERK and PI3K-Akt). Additionally, gene expression, neural proliferation, and activity may be regulated..$^{20}$ In this regard, glucose signaling pathways may have a pivotal role in memory function. ${ }^{19}$ Besides, neurotrophins and glucose metabolism mediators have similar intracellular signaling pathways. ${ }^{20}$

There seems to be an important relation between BDNF, IL- $1 \beta$, glucose, and corticosterone in the CNS, in such a way that cholinergic dysfunction in Alzheimer's disease (AD) pathophysiology is associated with their collective contribution. Given this significance, the scant literature on this topic is very surprising. Thus, one of the major goals in this study is understanding the probable relationship among the hippocampal BDNF, IL-1 $\beta$, corticosterone, and glucose levels. Although these mediators seem to be key mediators at the initial and developmental stages of neural damage, ${ }^{3,4,20}$ their impact on neurodegeneration have been studied less. The inflammatory mediators are often released as a consequence of neural injury and cause inflammation; however, the fate of other mediators in neurodegenerative disorders is not clearly understood. Hence, it is hypothesized here that nucleus basalis magnocellularis (NBM) injuries with the participant's increase or decrease of the above-mentioned mediators may affect other brain areas such as the hippocampus. ${ }^{3,7,16,21}$ Therefore, this study was designed to evaluate the levels of IL1- $\beta$ in the serum and hippocampus, as well as to measure the levels of hippocampal BDNF, corticosterone, and glucose after NBM lesion to verify the probability of such a relationship and/or its ensuing effects.

\section{Methods and Materials \\ Animals}

Thirty-six male Wistar rats (250-300 g) were kept under a $12 \mathrm{~h} \mathrm{light/dark} \mathrm{cycle} \mathrm{at} 22 \pm 2^{\circ} \mathrm{C}$ and with no particular restrictions on access to water and food. The subjects were randomly assigned to three equal groups (12 rats in each group): (1) control group, in which the rats were kept under similar conditions to the experimental group, but without any special intervention; (2) sham-operated group in which the animals received bilateral injections of saline (substance solvent) in NBM; and finally, (3) NBM-lesioned group, including the animals receiving bilateral injections of ibotenic acid. The animals in sham and NBM-lesioned groups were anesthetized by intraperitoneal injections of chloral hydrate $(450 \mathrm{mg} / \mathrm{kg})$ (Figure 1). Subsequently, the rats were immobilized on the stereotaxic apparatus (Stoelting, USA), and a small hole was made on either side of each skull. Ibotenic acid (10 $\mu \mathrm{g} / \mathrm{kg} ; 5 \mu \mathrm{g} / \mu \mathrm{L}$ for each side, Sigma) was injected at the $\mathrm{NBM}$ with coordination of anterior-posterior $(\mathrm{AP})=1.2$, mead line $(\mathrm{ML})= \pm 3.2 \mathrm{~mm}$, dorsal-ventral $(\mathrm{DV})=7.5$ $\mathrm{mm}$ from the skull surface. ${ }^{22}$ Also, normal saline 0.9\% (5 $\mu \mathrm{g} / \mu \mathrm{L}$ ) was injected bilaterally in the animals in the sham group.

\section{Behavioral Apparatus and Method}

The passive avoidance test was performed as our previously published data ${ }^{22}$ on 32 male rats (control, $\mathrm{n}=11$; sham, $\mathrm{n}=10$; and NBM-lesion, $\mathrm{n}=11$ ). The acquisition trial, retention trial, and electrical foot shocks were three phases of the test. Each rat was habituated to the apparatus for 300 seconds, 20 days after the lesion induction. On the next day (i.e., 21 days after NBM lesion), immediately after the darkroom stay, the rat received a single foot electric shock ( $50 \mathrm{~Hz}, 0.2 \mathrm{~mA}$ and $3 \mathrm{~s}$ ). The three retention trials were tested on 1, 7, and 21 days after receiving the foot shock. Accordingly, step-through latency (STL) and total time spent in the dark compartment (DS) were recorded.

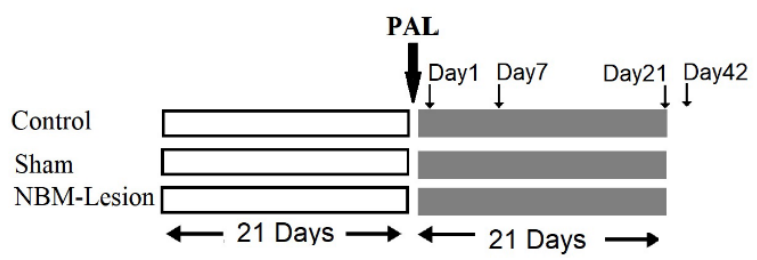

Figure 1. Experimental Protocol for Different Groups and the Days on Which Memory Acquisition and Retention Were Tested. 
The memory function was assessed by the remembrance of the received shock, that is the darkroom entrance latency and spending more time in the light room.

Estimation of Serum IL-1 $\beta$ and Hippocampal IL-1 $\beta$, BDNF, Corticosterone, and Glucose levels

In the next step, the animals were first anesthetized with an intraperitoneal injection of urethane $(1.5 \mathrm{~g} / \mathrm{kg})$ and then sacrificed. They were decapitated 42 days after the NBM lesion induction (i.e., 21 days after the behavioral test). The brains were instantly discarded from the skull; also, the trunk blood samples were centrifuged at the speed of $6000 \mathrm{rpm}$ for 20 minutes. The serum samples were collected and stored at $-80{ }^{\circ} \mathrm{C}$ until the time for the laboratory assays. To detect IL- $1 \beta$ levels in the hippocampus and serum, the commercial enzyme linked immunosorbent assay (ELISA) kits were used (Koma Biotech Cat\# K0331212, RRID: AB_2636820).

The hippocampus of each rat was immediately dissected on dry ice and placed in a ProblockTM-50, EDTA-free (Gold Bio Co., USA) phosphate-buffered solution (PBS, $\mathrm{pH}$ 7.4, $0.01 \mathrm{M}$ ) and complete protease inhibitor cocktail solution. Afterward, the hippocampus was homogenized and then centrifuged (cooled centrifuge at $4^{\circ} \mathrm{C}, 10000$ $\mathrm{g} / 20 \mathrm{~min}$ ). The samples were collected and kept at $-80^{\circ} \mathrm{C}$ for subsequent analysis. Hippocampal CORT, BDNF, and glucose levels were measured according to the methods supplied by the manufacturers' standard protocol of using ELISA kits, for BDNF (Promega Cat\# G7610, RRID: AB_2571723), Corticosterone (DRG Cat\# EIA4164, RRID: AB_2636819), and glucose oxidase method (Colorimetric Enzymatic; Pars Azmun Co., Tehran, Iran). Moreover, for the chemical analysis of serum and hippocampus, six samples were randomly selected from each group (i.e., 18 samples for serum and 18 samples for hippocampus analysis).

\section{Histology}

The approximate places of ibotenic acid injection are

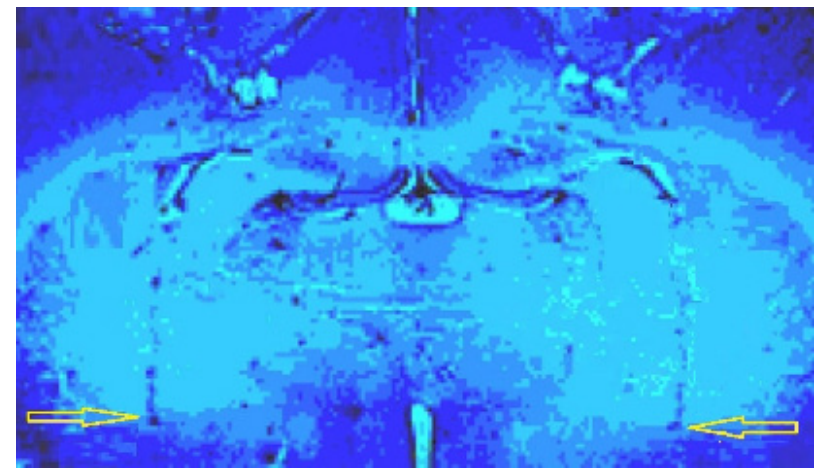

Figure 2. Position of Injection Cannula Tips in the NBM Regions of the Entorhinal Cortex for All the Rats Included in the Data Analyses. illustrated in Figure 2. The injection site was trodden on the brain section of the rat brain atlas. ${ }^{23}$. The formalinfixed brains were frozen, and serial transverse $60 \mu \mathrm{m}$ thick sections of the brain were cut to verify the ibotenic acid injection. Eventually, histological sections were observed at light microscopy.

\section{Data Analysis}

Total stay time in the darkroom was analyzed using the nonparametric Kruskal-Wallis test followed by a twotailed Mann-Whitney $U$ test.

The ANOVA analysis was used for Hippocampal BDNF, CORT, IL- $1 \beta$, and glucose data. Accordingly, posthoc Tukey's test was utilized for multiple comparisons of groups. Moreover, Pearson's correlation analysis and linear regression were applied to identify the correlation between variables (means \pm SEM, $P<0.05$ was significant).

\section{Results}

\section{NBM Lesion Induced Memory Deficit in Rats}

Our data analysis showed no significant differences between control and sham groups in the latency of day 1, 7, and 21 (Figure 3A). Thus, latency and memory functions were not altered by the surgery. However, drastic memory impairments were detected in the NBM-
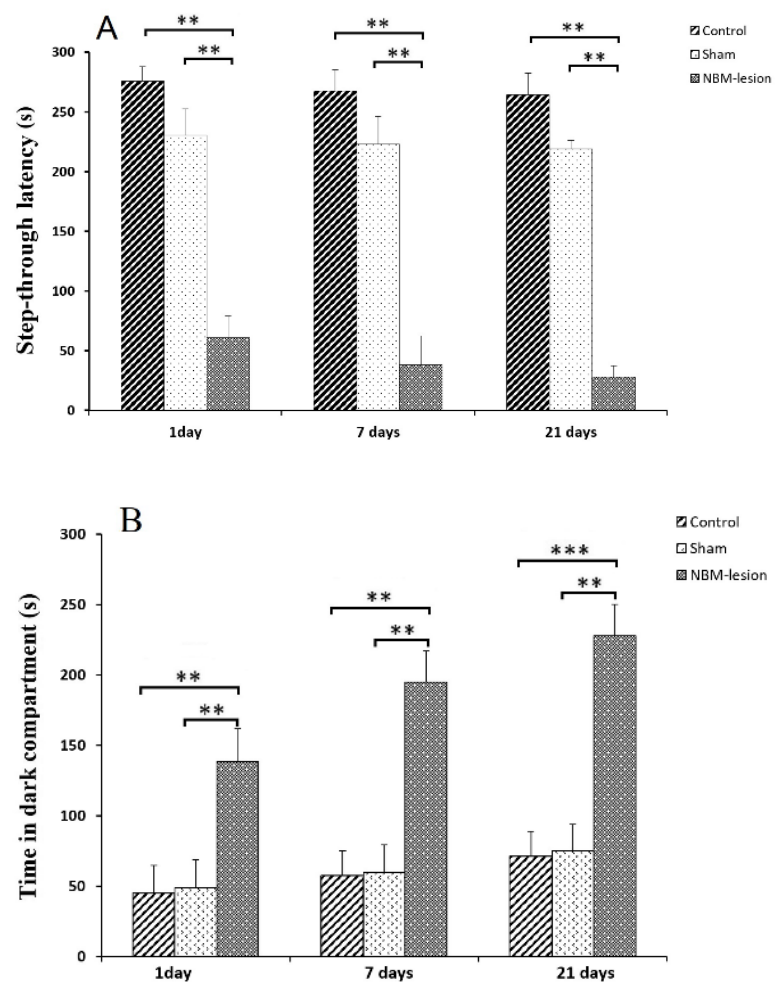

Figure 3. Effect of NBM Lesion in Step-Through Latency and Time in Dark Compartment of Passive Avoidance Test During Retention Test 7,1 , and 21 Days After Electrical Foot Shock Delivery in the Different Treatment Groups (Control: $n=11$; Sham: $n=10$; NBMlesion: $\mathrm{n}=10),{ }^{* *} P<0.01,{ }^{* * *} P<0.001$. 
lesioned group and, after foot shock, in the sham group (both, on days 1,7 , and $21, P<0.01$ ) compared to the control group. A lengthy latency was considered as a sign of memory improvement.

The result of the total time spending in DS revealed that the NBM-lesioned group had significant stay time enhancement a day after the foot shock (day 1) compared to the control and sham groups $(P<0.01$; Figure 3$)$. The analysis also indicated that the NBM lesion had interfered with memory retention. As illustrated in Figure 3, the DS values of the NBM-lesioned group on day 7 were significantly longer compared with other groups (control and sham, $P<0.01$ ). Moreover, the recorded DS on day 21 for the NBM-lesioned group showed significantly higher values compared to other ones (respectively, $P<0.001$ and $P<0.01$ for control and sham groups).

As previously mentioned, the total DS time was analyzed over three trials using relevant samples to evaluate the ingroup differences. Subsequently, the obtained values of each group were compared between pairs of days 1 and 7, days 7 and 21, and days 1 and 21 (Figure 4), in which the DS time of control and the sham groups showed no significant differences.

However, in a similar comparison, the NBM-lesioned group exhibited a significant difference $(P<0.05$; Figure $4)$. Nonetheless, the DS increased in all groups in later

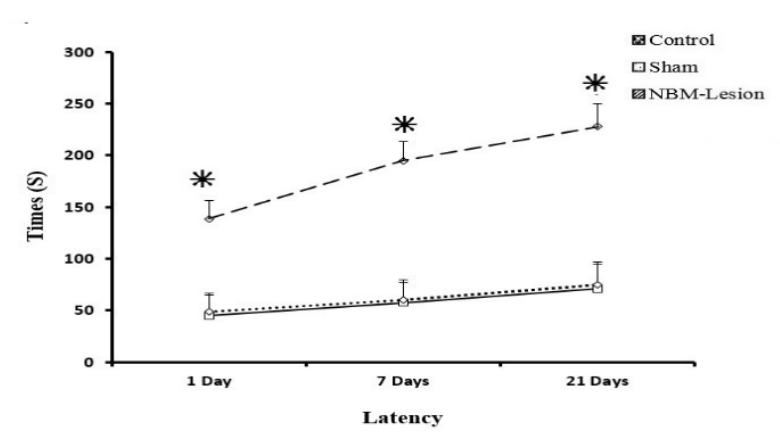

Figure 4. Trend Line of Time in Dark Compartment of Passive Avoidance Test After Electrical Foot Shock Delivery (Within Groups). (Control: $n=11$; Sham: $n=10$; NBM-lesion: $n=10$ ), $* P<0.05$
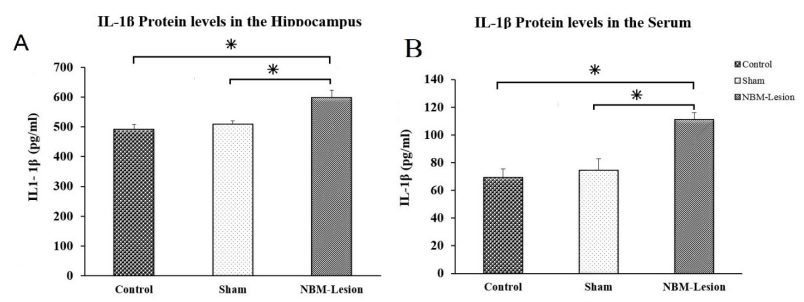

Figure 5. (A) Comparison of NBM-Lesion Effects on IL1- $\beta$ Protein Levels in the Hippocampus, (B) in the Serum $(\mathrm{pg} / \mathrm{mL})$ in the Different Groups $\left(\mathrm{n}=6\right.$, in each group); ${ }^{* *} P<0.01,{ }^{*} P<0.05$. days.

NBM Lesion Increased Hippocampal and Serum IL-1 $\beta$ Levels in Rats

The ANOVA and post-hoc Tukey's analysis showed no significant differences $(P>0.05)$ between control and sham hippocampal IL-1 $\beta$ levels; this indicated that surgery did not impact the hippocampal IL-1 $\beta$ levels (Figure 5).

Also, the hippocampal IL-1 $\beta$ levels increased significantly $(P<0.05)$ in the NBM-lesioned group compared to other groups (respectively, $P<0.01$ and $P<0.05$ in control and sham). Moreover, the serum IL$1 \beta$ levels did not show significant differences in the control and sham groups. However, there were significant differences between the NBM-lesioned group and other ones (Figure 5).

\section{NBM Lesion Decreased BDNF Levels in the Hippocampus of Rats}

No significant differences were observed in BDNF levels of the hippocampus between sham and control groups (ANOVA; Tukey's test; $P>0.05$ ), showing that surgery had no consequences on the BDNF levels after recovery (Figure 6A). However, a significant decrease was observed in the BDNF levels between NBM-lesioned and two other groups (respectively, $P<0.01$ and $P<0.05$ in control and sham groups). This indicates decreased levels of hippocampal BDNF in the NBM-lesioned rats.

\section{NBM Lesion Increased Glucose Levels in the Hippocampus of Rats}

Hippocampal glucose levels did not significantly differ in sham and control groups (ANOVA, Tukey's test, $P>0.05$ ). In Figure 6B, significant differences in the hippocampal glucose levels are observed between lesion, control, and sham groups $(P<0.05)$, indicating the enhancement of glucose levels in the NBM-lesioned rats.

\section{NBM Lesion Increased CORT Levels in the Hippocampus of Rats}

Based on the results of this study, hippocampal CORT levels did not differ significantly in the control or sham groups (ANOVA, Tukey's test, $P>0.05$ ). However, the hippocampal CORT levels were significantly different
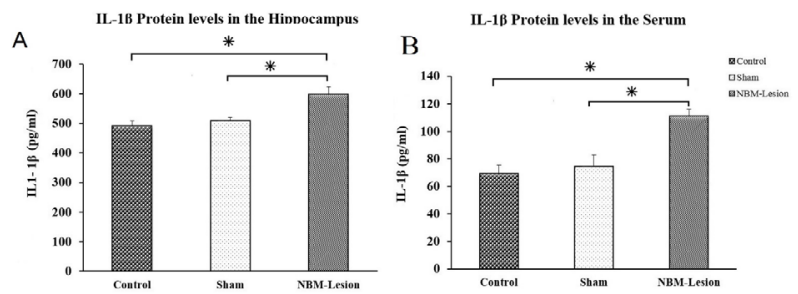

Figure 6. (A) Comparison of NBM-Lesion Effects on BDNF $(\mathrm{pg} / \mathrm{mL}) ;(B)$ Glucose $(\mathrm{mg} / \mathrm{dL})$; (C) CORT ( $\mathrm{nmol} / \mathrm{L})$ Levels in the Hippocampus. ( $\mathrm{n}=6$, in each group). ${ }^{* *} P<0.01,{ }^{*} P<0.05$. 
in the NBM-lesioned group compared to other groups (respectively, $P<0.01$ and $P<0.05$ in control and sham groups; Figure 6C).

\section{Correlation Between Hippocampal IL-1 $\beta$ and Serum IL-} $1 \beta$ Level

Our data displayed positive significant correlations between hippocampus IL- $1 \beta$ and serum IL- $1 \beta$ levels in all experimental groups (Pearson's analysis); correspondingly, the observed correlations were measured in the control group ( $\mathrm{r}=0.835, P<0.05)$, sham $(\mathrm{r}=0.851, P<0.05)$ and NBM-lesioned $(r=0.889, P<0.01)$ groups (Figure $7 \mathrm{~A})$.

\section{Correlations Between Hippocampal IL-1 $\beta$ and BDNF, Glucose, and CORT Levels}

Similarly, the findings showed significant negative correlations between hippocampal IL-1 $\beta$ and BDNF levels (Pearson's analysis); the observed correlations were measured in the control $(\mathrm{r}=-894, P<0.05)$, sham $(\mathrm{r}=-$ $0.870, P<0.05)$ and NBM-lesioned $(\mathrm{r}=-0.847, P<0.05)$ groups (Figure $7 \mathrm{~B}$ ).

Additionally, there were significant positive correlations between the hippocampal IL-1 $\beta$ and glucose levels in all groups (Figure $7 \mathrm{C}$ ), there were measured in the control group $(\mathrm{r}=0.853, \mathrm{P}<0.05)$, sham $(\mathrm{r}=0.880, P<0.05)$ and lesion $(r=0.820, P<0.05)$ groups.

Eventually, the statistical analyses demonstrated that hippocampal IL-1 $\beta$ levels significantly correlated with CORT levels in control, sham, and NBM-lesioned groups; these correlations were measured in the control group $(\mathrm{r}=0.866, P<0.05)$, sham $(\mathrm{r}=0.835, P<0.05)$ and lesion $(\mathrm{r}=0.874, P<0.05)$ groups (Figure $7 \mathrm{D})$.

\section{Discussion}

Based on our findings, the STL decreased and the DS time increased notably in the NBM-lesioned group on days 1,7 , and 21 after receiving foot shock (Figures 3-4). The decreased STL and increased DS in this group may have resulted from the disruption of the basal forebrain projections to other brain areas, such as the hippocampus, causing retention impairments. Neurodegeneration affects these aspects of memory deficits in numerous neuropsychological disorders. ${ }^{1}$ No adaptations were observed in the memory retention on days 1,7 , and 21 after receiving foot shock.

These findings propose an increase of the central and peripheral IL-1 $\beta$ levels in the NBM-lesioned group; This suggests that neurodegeneration of basal forebrain projection to the hippocampus may be initiated by inflammatory responses. Cytokines have a wide role in initiation, augmentation, continuation, and elimination of the inflammatory and immune responses, which makes them key mediators for the tissue response to damage.

There is evidence of low IL- $1 \beta$ levels in healthy brains $^{24}$ and high IL-1 $\beta$ levels subsequent to peripheral

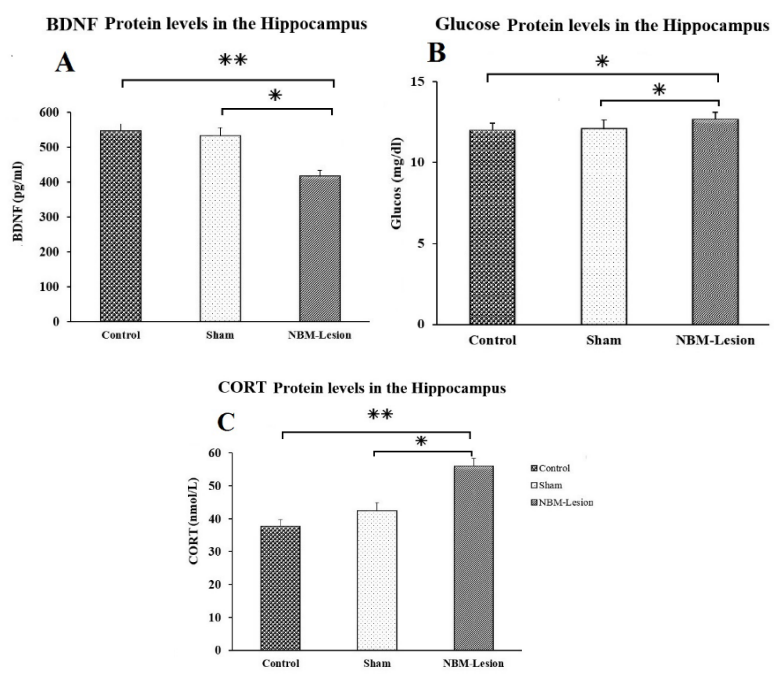

Figure 7. Linear Correlation Between; (A) Hippocampal IL1- $\beta$ and Serum IL1- $\beta$ Levels; (B) Hippocampal IL1- $\beta$ and BDNF Levels; (C) Hippocampal IL1- $\beta$ and Glucose Levels; (D) Hippocampal IL1- $\beta$ and Corticosterone (CORT) Levels in Different Groups, $(n=6$, in each group). A significant positive correlation between hippocampal and serum IL1- $\beta$ levels. A positive correlation between hippocampal and serum IL1- $\beta$ (Control; $R=0.835$, Sham; $\mathrm{R}=0.85$ and lesion; $\mathrm{R}=0.889$ groups), hippocampal IL$1 \beta$ and Glucose levels (Control; $R=0.853$, Sham; $R=0.880$ and lesion; $R=0.820$ groups) and hippocampal IL- $1 \beta$ and CORT levels (Control; $R=0.866$, Sham; $R=0.835$ and lesion; $R=0.847$ groups) was found. A negative correlation between hippocampal IL- $1 \beta$ and BDNF levels (Control; $R=0.866-$, Sham; $R=0.870$ and lesion; $R=-0.847$ groups) was found. $C O R T=$ Corticosterone, $B D N F=$ Brain Derived Neurotrophic Factor, IL- $1 \beta=$ Interleukin$1 \beta$.

infections, surgery, brain injury, and social isolation. ${ }^{25,26}$ Also, the hippocampus has been proved to be the main place related to learning and memory. Therefore, it is reasonable to consider it as a predominant location of many memory-related neurotransmitters or neuromodulators receptors, such as IL-1 receptors. ${ }^{13}$ Similarly, the intracerebroventricular (i.c.v.) injection and bilateral intra-hippocampal injection of IL-1 $\beta$ (10 or $20 \mathrm{ng}$ ) impaired hippocampal fear conditioning in healthy animals. ${ }^{26}$ As a result, the NBM lesion stimulated hippocampal IL-1 $\beta$ secretion and facilitated memory impairment. However, pieces of evidence either show no impact on memory processes, or present lucrative effects of IL- $1 \beta$ on it. ${ }^{27}$

IL- $1 \beta$ has been identified as a key mediator in neuroendocrine and neurobehavioral stress responses. Moreover, it has been shown that IL-1 $\beta$ stimulates the secretion of corticotrophin-releasing factor in the hypothalamus. $^{28}$

The involvement of the hippocampus and its basal forebrain cholinergic innervation in stress responses is regulated by the hypothalamus-pituitary-adrenal (HPA) axis. ${ }^{3}$ Studies demonstrated that stress, either the acute 
or inescapable one, is accompanied by the increased release of acetylcholine and regulates the acetylcholine availability in the prefrontal cortex and hippocampus. Also, this report emphasized the stress regulation via the involvement of the cholinergic system. ${ }^{29,30}$

The glucocorticoid, that are released under acute/ chronic stress conditions affect the cognitive functions in the hippocampus and prefrontal cortex. ${ }^{31}$ As such, the hippocampus is introduced with a particular role in glucocorticoid hormone signaling, together with help of the HPA axis contributes to cognitive function modulation. ${ }^{2}$

The available evidence suggests that non-physiological levels of glucocorticoids can harm the hippocampal neurons and decrease dendrite complexity. ${ }^{32}$ Other studies on animals have highlighted the role of neurodegeneration in glucocorticoid levels of the brain. As such, the targeted lesions of cholinergic input to the hippocampus were shown to result in the HPA axis impairment and reduced hippocampal glucocorticoid receptors. Moreover, circulating corticosterone values did not represent any relation with the decrease in glucocorticoid receptor mRNA. ${ }^{32,33}$ Hence, the hippocampal innervation through cholinergic neurons may normally take part in the modulation of glucocorticoid receptors and HPA functions.

In brief, the data suggest that neural involvement through neuroendocrine and transcriptional signaling pathways and other mechanisms, which could lead to corticosterone upregulation (following the basal forebrain dysfunction), could consequently develop the impairment of neural functions and plasticity. ${ }^{32,33}$ In some reports, it is indicated that high levels of CORT over a prolonged period of time can cause neurodegeneration in the brain; moreover, it may lead to severe cognition impairment due to the decrease of choline acetyltransferase and acetylcholinesterase functions in the hippocampus. ${ }^{32,33}$ Also, glucocorticoids can prevent the storage and acetylcholine activity, by strengthening the cholinesterase activity. ${ }^{34}$

Subsequent studies proposed the hippocampal protein kinase A (PKA) signaling alteration with cholinergic input elimination. ${ }^{35}$ They documented that intensification of PKA signaling activity was along with pro-inflammatory gene expression such as inducible nitric oxide synthase and cyclooxygenase- 2 in the hippocampus. ${ }^{36}$

Normally, a high density of glucocorticosteroid receptors has been reported in the mammalian hippocampus that can associate to negative feedback regulation of the HPA axis by release of glucocorticosteroids. ${ }^{37}$ Therefore, the hippocampal neurons, with a crucial function in cognition, are specifically sensitive to injury after a singledose injection of CORT; this leads to the impairment of spatial memory and synaptic plasticity. ${ }^{38,39}$ As mentioned before, both the glucocorticoids and cholinergic system have key functions in learning and memory.

The considerable role of stress in memory and mental disorders has been indicated in other studies too. ${ }^{40}$ CORT is a key stress mediator that affects memory. In this study, the increase of CORT levels in the NBM-lesioned group was suggested as an effective factor for the IL$1 \beta$ increment in stressed rats. Moreover, these findings showed that circulating corticosterone levels correlated with the hippocampal IL- $1 \beta$ concentration in stressed rats. ${ }^{41}$ Therefore, the reciprocal and severe effects of corticosterone, BDNF, and IL- $1 \beta$ on the hippocampusdependent memory in stress and neurodegeneration could be proposed. The results of this study indicate that NBM lesion after 42 days was associated with the decrease of BDNF values in the hippocampus. Recently, it has been proposed that HPA axis hyperactivity or a considerable increase of glucocorticoids, following the exposure to chronic CORT, may have regulatory effects on BDNF and CREB expressions.

On one hand, chronic CORT may induce HPA axis hyperactivity or excessive increase of glucocorticoid levels that regulate the expression of BDNF and CREB, thus affecting the BDNF pathway functions in the hippocampus. ${ }^{42}$ On the other hand, the low expression value of BDNF and CREB may involve in the HPA axis dysfunction. ${ }^{43}$ BDNF is synthesized in inflammatory cells, fibroblasts, and other cell types. ${ }^{44}$ Moreover, some studies have suggested a correlation between central and peripheral BDNF levels, hence, concluding that BDNF can be cross the blood-brain barrier. Nevertheless, other studies could not find such a correlation; also they revealed that neurotrophins did not cross the blood-brain barrier. ${ }^{45}$

In our previously published study, an increase of hippocampal corticosterone levels and a decrease of BDNF ones were shown in stressed rats. ${ }^{41}$ However, the BDNF levels were not followed by the IL- $1 \beta$ increase in these animals. This contradiction may be due to the synergistic inhibitory effects of IL- $1 \beta$ and corticosterone on BDNF production after the neurodegeneration. According to a recent report, IL-1 $\beta$ interferes with the intrinsic neurotrophic support by interrupting the BDNF signaling cascade; this mechanism has been proposed to be linked to the progression of memory impairments in some chronic diseases, such as AD. ${ }^{8}$ Besides, some studies proposed the role of corticosterone in suppressing the BDNF expression at the mRNA and its production. ${ }^{46}$ Moreover, exposure to stress or high doses of glucocorticoids depressed neurogenesis and decreased BDNF levels. ${ }^{47}$

Neurotrophins, such as BDNF, act as critical modulators in the neural development, survival, and functions of the nervous system. ${ }^{48}$ Therefore, impairment of the BDNF signaling pathways under some conditions can lead to neural dysfunction and neurodegenerative disease 
symptoms, such as learning and memory deficits.

Available pieces of evidence proposed that also metabolic alterations play an important role in the initiation and progression of neurodegenerative disorders, such as Alzheimer's and Parkinson's diseases; besides, glucose utilization decreased in certain areas of these patients' brains. ${ }^{49}$ Moreover, proper glucose metabolism leads to maintaining the healthy functioning of neurons and other cells, mitochondrial energy production, DNA repair, neurotrophic factor signaling, and neuronal plasticity. ${ }^{49}$ Although glucose has been proposed to influence memory function, it has more impact on other cognitive functions by synthesizing acetylcholine and insulin and their consequent effects on the hippocampal performance..$^{50}$

Our results showed that hippocampal glucose concentration increased after NBM-lesion. However, some studies have proposed that low doses of central and peripheral IL-1 $\beta$ administration caused low glucose levels in the blood and hypothalamus. They claimed that treatments affected the set point for glucose homeostasis through the regulatory neuroendocrine mechanisms of the $\mathrm{CNS}^{51}$; whereas, the present study observed synchronous increase of glucose, CORT, and IL- $1 \beta$, plus the decrease of BDNF concentrations in the hippocampus of NBMlesioned rats. The discrepancy between our findings and the results of other studies on glucose levels might be related to methodology differences. The exogenous administration of IL- $1 \beta$, BDNF, or CORT seems to have resulted in low glucose levels, while neuroinflammation, followed by neurodegeneration, has led to an increase in IL- $1 \beta$ and CORT, plus a decrease in BDNF concentrations of the brain, hence, resulting in central hyperglycemia. All in all, these findings propose a vigorous cycle of neurodegeneration along with the subsequent cascade of events that can alter inflammatory and neuroprotective metabolisms, as well as the neuroendocrine stress-related responses.

In this study, the present variables related to the hippocampal IL-1 $\beta$, glucose, BDNF, corticosterone and serum IL-1 $\beta$ concentrations (Figures 4-6) after NBM lesions were coupled with our formerly published data on memory retrieval impairment recorded 21 days after a foot shock in the NBM-lesioned rats; this comparison provided strong evidence in favor of our hypothesis concerning a causal relationship of these parameters.

Animal studies have suggested that central and peripheral inflammations are important factors in the damage and repair of the brain. ${ }^{6}$ Also, our results showed higher concentrations of IL- $1 \beta$ in the hippocampus compared to the serum. Additionally, the serum and hippocampal IL-1 $\beta$ were directly and significantly correlated within these groups (Figure 7). In previous studies, systemic inflammation has been shown to affect the brain and its functions. However, our result revealed the ability of hippocampal IL- $1 \beta$ for cross the blood-brain barrier, resulting in the probable systemic effects of the central neuroinflammation over time, interlinking to the probable systemic effects of central neuroinflammation over time.

In conclusion, our findings demonstrated a significant positive correlation among hippocampal IL-1 $\beta$, corticosterone, and glucose, as well as a major negative correlation between IL-1 $\beta$ and BDNF in NBM-lesioned rats (Figure 6).

According to these findings, higher levels of IL- $1 \beta$ in the hippocampus after NBM lesion is partly induced through an inflammatory pathway. However, the stress-related biological pathways and brain metabolic dysfunction along with decreased BDNF levels may be responsible for the symptoms of some neurodegenerative diseases. Further investigations about these biological pathways are required for future studies. Therefore, considering a complex set of problems for managing neurodegenerative diseases is reasonable. Alteration of these factors will result in a chain of events and problems over time. Thus, establishing a molecular basis underlying the effect of our findings and the beginning time of these events is necessary.

\section{Conflict of Interest}

The authors declare that they have no conflict of interests.

\section{Acknowledgments}

The authors would like to thank Dr. Mehdi Hedayati, associate professor of Biochemistry at the Research Institute for Endocrine Sciences, Shahid Beheshti University of Medical Sciences, for his valuable assistance.

\section{Authors' Contribution}

$\mathrm{NH}$ and HA: study design, $\mathrm{NH}$ and MR: perform experiment, data gathering and analysis, manuscript preparation, $\mathrm{SN}$ : language editing, All of Author's read and approved manuscript

\section{Funding/Support}

This research did not receive any specific grant from funding agencies in the public, commercial, or not-for-profit sectors.

\section{Ethical Statement}

The experimental protocols were authorized by the Ethical Committee of Isfahan University of Medical Sciences (IR.MUI. REC. 389121) and organized in accordance with the Helsinki Declaration (1989 Revision).

\section{References}

1. Ballinger EC, Ananth M, Talmage DA, Role LW. Basal forebrain cholinergic circuits and signaling in cognition and cognitive decline. Neuron. 2016;91(6):1199-218. doi: 10.1016/j. neuron.2016.09.006

2. Vedhara K, Hyde J, Gilchrist ID, Tytherleigh M, Plummer S. Acute stress, memory, attention and cortisol. Psychoneuroendocrinology. 2000;25(6):535-49. doi: 10.1016/s0306-4530(00)00008-1.

3. Kim EJ, Pellman B, Kim JJ. Stress effects on the hippocampus: a 
critical review. Learn Mem. 2015;22(9):411-6. doi: 10.1101/ Im.037291.114.

4. Cañavate M, García de Galdeano A, Arteaga O, Montalvo $H$, Revuelta $M$, Hilario $E$, et al. Cross-talk between nervous and immune systems: cytokines modulating morphology and function of both systems under stress conditions. Microscopy: Advances in Scientific Research and Education. 2014;1:41421.

5. de Quervain DJ, Roozendaal B, McGaugh JL. Stress and glucocorticoids impair retrieval of long-term spatial memory. Nature. 1998;394(6695):787-90. doi: 10.1038/29542.

6. French RA, VanHoy RW, Chizzonite R, Zachary JF, Dantzer $R$, Parnet $P$, et al. Expression and localization of p80 and p68 interleukin-1 receptor proteins in the brain of adult mice. J Neuroimmunol. 1999;93(1-2):194-202. doi: 10.1016/s01655728(98)00224-0.

7. Rachal Pugh C, Fleshner M, Watkins LR, Maier SF, Rudy JW. The immune system and memory consolidation: a role for the cytokine IL-1beta. Neurosci Biobehav Rev. 2001;25(1):29-41. doi: 10.1016/s0149-7634(00)00048-8.

8. Lee JW, Lee YK, Yuk DY, Choi DY, Ban SB, Oh KW, et al. Neuro-inflammation induced by lipopolysaccharide causes cognitive impairment through enhancement of betaamyloid generation. J Neuroinflammation. 2008;5:37. doi: 10.1186/1742-2094-5-37.

9. Zhao G, Dharmadhikari G, Maedler K, Meyer-Hermann M. Possible role of interleukin-1 $\beta$ in type 2 diabetes onset and implications for anti-inflammatory therapy strategies. PLoS Comput Biol. 2014;10(8):e1003798. doi: 10.1371/journal. pcbi.1003798.

10. Vounotrypidis P, Kouklakis G, Anagnostopoulos K, Zezos P, Polychronidis A, Maltezos E, et al. Interleukin-1 associations in inflammatory bowel disease and the enteropathic seronegative spondylarthritis. Auto Immun Highlights. 2013;4(3):87-94. doi: 10.1007/s13317-013-0049-4.

11. Liao Z, Xiao HT, Zhang Y, Tong RS, Zhang LJ, Bian Y, et al. IL-1 $\beta$ : a key modulator in asthmatic airway smooth muscle hyper-reactivity. Expert Rev Respir Med. 2015;9(4):429-36. doi: 10.1586/17476348.2015.1063422.

12. Kay J, Calabrese L. The role of interleukin- 1 in the pathogenesis of rheumatoid arthritis. Rheumatology (Oxford). 2004;43 Suppl 3:iii2-iii9. doi: 10.1093/rheumatology/keh201.

13. Gonzalez P, Machado I, Vilcaes A, Caruso C, Roth GA, Schiöth $\mathrm{H}$, et al. Molecular mechanisms involved in interleukin 1-beta (IL-1 $\beta$ )-induced memory impairment. Modulation by alphamelanocyte-stimulating hormone $(\alpha-M S H)$. Brain Behav Immun. 2013;34:141-50. doi: 10.1016/j.bbi.2013.08.007.

14. Goshen I, Yirmiya R. Interleukin-1 (IL-1): a central regulator of stress responses. Front Neuroendocrinol. 2009;30(1):30-45. doi: 10.1016/j.yfrne.2008.10.001.

15. Tong L, Prieto GA, Kramár EA, Smith ED, Cribbs DH, Lynch G, et al. Brain-derived neurotrophic factor-dependent synaptic plasticity is suppressed by interleukin-1 $\beta$ via p38 mitogenactivated protein kinase. J Neurosci. 2012;32(49):17714-24. doi: 10.1523/jneurosci.1253-12.2012.

16. Tyler WJ, Alonso M, Bramham CR, Pozzo-Miller LD. From acquisition to consolidation: on the role of brain-derived neurotrophic factor signaling in hippocampal-dependent learning. Learn Mem. 2002;9(5):224-37. doi: 10.1101/ Im.51202.

17. Lu Y, Christian K, Lu B. BDNF: a key regulator for protein synthesis-dependent LTP and long-term memory?
Neurobiol Learn Mem. 2008;89(3):312-23. doi: 10.1016/j. nlm.2007.08.018.

18. Heldt SA, Stanek L, Chhatwal JP, Ressler KJ. Hippocampusspecific deletion of BDNF in adult mice impairs spatial memory and extinction of aversive memories. Mol Psychiatry. 2007;12(7):656-70. doi: 10.1038/sj.mp.4001957.

19. Lee HJ, Seo HI, Cha HY, Yang YJ, Kwon SH, Yang SJ. Diabetes and Alzheimer's disease: mechanisms and nutritional aspects. Clin Nutr Res. 2018;7(4):229-40. doi: 10.7762/ cnr.2018.7.4.229.

20. Mainardi M, Fusco S, Grassi C. Modulation of hippocampal neural plasticity by glucose-related signaling. Neural Plast. 2015;2015:657928. doi: 10.1155/2015/657928.

21. Silva-Adaya D, Pérez-De La Cruz V, Herrera-Mundo MN, Mendoza-Macedo K, Villeda-Hernández J, Binienda Z, et al. Excitotoxic damage, disrupted energy metabolism, and oxidative stress in the rat brain: antioxidant and neuroprotective effects of L-carnitine. J Neurochem. 2008;105(3):677-89. doi: 10.1111/j.1471-4159.2007.05174.x.

22. Hosseini N, Alaei H, Reisi P, Radahmadi M. The effect of treadmill running on memory before and after the NBM-lesion in rats. J Bodyw Mov Ther. 2013;17(4):423-9. doi: 10.1016/j. jbmt.2012.12.005.

23. Paxinos G, Watson C. The Rat Brain in Stereotaxic Coordinates: Hard Cover Edition. Elsevier; 2006.

24. Vitkovic L, Bockaert J, Jacque C. "Inflammatory" cytokines: neuromodulators in normal brain? J Neurochem. 2000;74(2):457-71. doi: 10.1046/j.14714159.2000.740457.x.

25. Eriksson C, Van Dam AM, Lucassen PJ, Bol JG, Winblad B, Schultzberg M. Immunohistochemical localization of interleukin-1beta, interleukin-1 receptor antagonist and interleukin-1 beta converting enzyme/caspase- 1 in the rat brain after peripheral administration of kainic acid. Neuroscience. 1999;93(3):915-30. doi: 10.1016/s0306-4522(99)00178-5.

26. Pugh CR, Nguyen KT, Gonyea JL, Fleshner M, Wakins LR, Maier SF, et al. Role of interleukin-1 beta in impairment of contextual fear conditioning caused by social isolation. Behav Brain Res. 1999;106(1-2):109-18. doi: 10.1016/s01664328(99)00098-4.

27. Goshen I, Kreisel T, Ounallah-Saad H, Renbaum P, Zalzstein Y, Ben-Hur T, et al. A dual role for interleukin-1 in hippocampal-dependent memory processes. Psychoneuroendocrinology. 2007;32(8-10):1106-15. doi: 10.1016/j.psyneuen.2007.09.004.

28. Zelazowski P, Patchev VK, Zelazowska EB, Chrousos GP, Gold PW, Sternberg EM. Release of hypothalamic corticotropinreleasing hormone and arginine-vasopressin by interleukin 1 beta and alpha MSH: studies in rats with different susceptibility to inflammatory disease. Brain Res. 1993;631(1):22-6. doi: 10.1016/0006-8993(93)91181-q.

29. Mark GP, Rada PV, Shors TJ. Inescapable stress enhances extracellular acetylcholine in the rat hippocampus and prefrontal cortex but not the nucleus accumbens or amygdala. Neuroscience. 1996;74(3):767-74. doi: 10.1016/03064522(96)00211-4.

30. Kaufer D, Friedman A, Seidman S, Soreq H. Acute stress facilitates long-lasting changes in cholinergic gene expression. Nature. 1998;393(6683):373-7. doi: 10.1038/30741.

31. Popoli M, Yan Z, McEwen BS, Sanacora G. The stressed synapse: the impact of stress and glucocorticoids on glutamate transmission. Nat Rev Neurosci. 2011;13(1):22-37. 
doi: 10.1038/nrn3138.

32. Han JS, Bizon JL, Chun HJ, Maus CE, Gallagher M. Decreased glucocorticoid receptor mRNA and dysfunction of HPA axis in rats after removal of the cholinergic innervation to hippocampus. Eur J Neurosci. 2002;16(7):1399-404. doi: 10.1046/j.1460-9568.2002.02191.x.

33. Helm KA, Han JS, Gallagher M. Effects of cholinergic lesions produced by infusions of 192 IgG-saporin on glucocorticoid receptor mRNA expression in hippocampus and medial prefrontal cortex of the rat. Neuroscience. 2002;115(3):76574. doi: 10.1016/s0306-4522(02)00487-6.

34. Lee B, Sur BJ, Kwon S, Jung E, Shim I, Lee H, et al. Acupuncture stimulation alleviates corticosterone-induced impairments of spatial memory and cholinergic neurons in rats. Evid Based Complement Alternat Med. 2012;2012:670536. doi: 10.1155/2012/670536.

35. Lim CS, Kim YJ, Hwang YK, Bañuelos C, Bizon JL, Han JS. Decreased interactions in protein kinase A-glucocorticoid receptor signaling in the hippocampus after selective removal of the basal forebrain cholinergic input. Hippocampus. 2012;22(3):455-65. doi: 10.1002/hipo.20912.

36. Lee SY, Cho WH, Lee YS, Han JS. Impact of chronic stress on the spatial learning and GR-PKAc-NF-KB signaling in the hippocampus and cortex in rats following cholinergic depletion. Mol Neurobiol. 2018;55(5):3976-89. doi: 10.1007/ s12035-017-0620-5.

37. Belanoff JK, Gross K, Yager A, Schatzberg AF. Corticosteroids and cognition. J Psychiatr Res. 2001;35(3):127-45. doi: 10.1016/s0022-3956(01)00018-8.

38. Woolley CS, Gould E, McEwen BS. Exposure to excess glucocorticoids alters dendritic morphology of adult hippocampal pyramidal neurons. Brain Res. 1990;531(12):225-31. doi: 10.1016/0006-8993(90)90778-a.

39. Luine VN, Spencer RL, McEwen BS. Effects of chronic corticosterone ingestion on spatial memory performance and hippocampal serotonergic function. Brain Res. 1993;616(12):65-70. doi: 10.1016/0006-8993(93)90193-q.

40. Vogel S, Schwabe L. Learning and memory under stress: implications for the classroom. NPJ Sci Learn. 2016;1:16011. doi: 10.1038/npjscilearn.2016.11.

41. Radahmadi M, Alaei H, Sharifi MR, Hosseini N. Effects of different timing of stress on corticosterone, BDNF and memory in male rats. Physiol Behav. 2015;139:459-67. doi: 10.1016/j.physbeh.2014.12.004.
42. Mizuno M, Yamada K, Maekawa N, Saito K, Seishima M, Nabeshima T. CREB phosphorylation as a molecular marker of memory processing in the hippocampus for spatial learning. Behav Brain Res. 2002;133(2):135-41. doi: 10.1016/s01664328(01)00470-3.

43. Kunugi $\mathrm{H}$, Hori $\mathrm{H}$, Adachi $\mathrm{N}$, Numakawa T. Interface between hypothalamic-pituitary-adrenal axis and brain-derived neurotrophic factor in depression. Psychiatry Clin Neurosci. 2010;64(5):447-59. doi: 10.1111/j.1440-1819.2010.02135.x.

44. Grimsholm O, Rantapää-Dahlqvist $S$, Dalén T, Forsgren $S$. BDNF in RA: downregulated in plasma following anti-TNF treatment but no correlation with inflammatory parameters. Clin Rheumatol. 2008;27(10):1289-97. doi: 10.1007/s10067008-0910-4.

45. Etnier JL, Wideman L, Labban JD, Piepmeier AT, Pendleton DM, Dvorak KK, et al. The effects of acute exercise on memory and brain-derived neurotrophic factor (BDNF). J Sport Exerc Psychol. 2016;38(4):331-40. doi: 10.1123/jsep.2015-0335.

46. Schaaf MJ, De Kloet ER, Vreugdenhil E. Corticosterone effects on BDNF expression in the hippocampus. Implications for memory formation. Stress. 2000;3(3):201-8. doi: 10.3109/10253890009001124.

47. Hueston CM, Cryan JF, Nolan YM. Stress and adolescent hippocampal neurogenesis: diet and exercise as cognitive modulators. Transl Psychiatry. 2017;7(4):e1081. doi: 10.1038/ tp.2017.48.

48. Barrientos RM, Sprunger DB, Campeau S, Watkins LR, Rudy JW, Maier SF. BDNF mRNA expression in rat hippocampus following contextual learning is blocked by intrahippocampal IL-1beta administration. J Neuroimmunol. 2004;155(12):119-26. doi: 10.1016/j.jneuroim.2004.06.009.

49. Camandola S, Mattson MP. Brain metabolism in health, aging, and neurodegeneration. EMBO J. 2017;36(11):1474-92. doi: 10.15252/embj.201695810.

50. Scholey A, Camfield D, Macpherson H, Owen L, Nguyen $\mathrm{P}$, Stough $\mathrm{C}$, et al. Hippocampal involvement in glucose facilitation of recognition memory: event-related potential components in a dual-task paradigm. Nutr Aging. 2015;3(1):920. doi: 10.3233/nua-140042.

51. Del Rey A, Roggero E, Randolf A, Mahuad C, McCann S, Rettori V, et al. IL-1 resets glucose homeostasis at central levels. Proc Natl Acad Sci U S A. 2006;103(43):16039-44. doi: 10.1073/pnas.0607076103. 